\begin{tabular}{c} 
International Journal of Engineering \& Technology, 7 (3) (2018) 1605-1608 \\
International Journal of Engineering \& Technology \\
SPC \\
Website: $\begin{array}{c}\text { www. sciencepubco.com/index.php/IJET } \\
\text { doi: } 10.14419 / \text { ijet. } 7 \text { is.15518 } \\
\text { Research paper }\end{array}$ \\
\hline
\end{tabular}

\title{
Design of rogowski coil to measure the power frequency alternating current
}

\author{
A. Benitto ${ }^{1}$, V. Indragandhi ${ }^{2}$, R. Abarna ${ }^{1}$, R. Raja Singh ${ }^{2}$ \\ ${ }^{1}$ PG Scholar, Thiagarajar College of Engineering, Madurai \\ ${ }^{2}$ Associate Professor, Vellore Institute of Technology, Vellore \\ *Corresponding author E-mail: arunindra08@gmail.com
}

\begin{abstract}
For the growth of high voltage transmission power cables, it is necessary to calculate the current and voltage values. Current Transformers (CT) are used to convert the larger current values into smaller values. CT develop the convenient way of measurement in an alternating current transmission line. If CT becomes saturated then secondary current in a CT gets distorted because of a larger primary current. This distorted secondary current leads to a malfunction in the measurement side. So, detection and correction of the distorted current caused by the saturated CT becomes essential. To avoid these problems, we can use Rogowski Coil (RC) to measure the alternating current.

$\mathrm{RC}$ is a toroidal coil used for measuring alternating current without touching the conductor. A RC is an evenly wound coil of $\mathrm{N}$ turns per meter on a nonmagnetic core of constant cross-sectional area A. Normally an alternating current produces a magnetic field when current flows through a conductor. $\mathrm{RC}$ is used to sense the magnetic field and rise to an induced voltage within the coil which is proportional to the rate of change of the current being measured. This RC current measurement is based on adopted reconstruction methods. In this paper, use two reconstructions methods to measure the alternating current. They are Analog integrator and Numerical Differentiation (ND). These reconstruction methods providing accurate as well as reduced time for correctly tracking the measured current.
\end{abstract}

Keywords: Flexible; Coil; Mutual Inductance; Electro Motive Force; Reconstruction; Alternating Current; Integrator.

\section{Introduction}

$\mathrm{CT}$ is one of the best devices to measure the AC. In CT, current in the secondary winding which is proportional to the current flowing through the primary winding. Generally, CTs are used to convert the high current from electrical circuit into measurable current range. They provide isolation to the ammeters and other measuring instruments. So, the detection and correction of CT saturation could substantially improve the performance [1-2].

$\mathrm{RC}$ is to overcome the power measurement problems and CT drawbacks. $\mathrm{RC}$ is an alternative transducer of CT. The inventor of this RC is German Physicist Walter Rogowski. So, it is called as RC. RC is a circular coil used for measuring alternating currents. $\mathrm{RC}$ is a nonmagnetic toroidal coil with $\mathrm{N}$ number of turns per meter on a non-magnetic former of constant cross-sectional area in which is placed around the conductor. The alternating magnetic field produced by the current induces a voltage in the coil which is proportional to the rate of change of alternating current flows through the conductor [3-4]. In construction side, the starting point of the winding wire is connected to the central axis of the former. Another end of the coil is connected to the integrator circuit. And in ND Reconstruction method, coil is connected to the LabVIEW software through DAQ card. The coil is to fix around the conductor and to join the free ends of the coil. Not only, the RC is useful in conventional applications, but also, there are other cases where the RC is the best choice. Nongeometric structure and capability in measuring high amplitude current and etc. It has various applications in testing laboratories, industrial measurement instruments, and unconventional measuring systems. It is also useful in triggering applications.

\subsection{Problem description}

Mainly RC is used instead of CT applications. Amplifying the output signals magnifies the associated noise signals. This was a real challenge for high-frequency applications. The accuracy of current measurement using RC is dependent mainly on the adopted reconstruction method. Common Reconstruction Techniques are Analog integrator, Numerical Integration (NI), Numerical Integration Substitution (NIS), Transfer Function (TF) and Fitted Inverse Transfer Function (FITF), Numerical Differentiation [5-6]. NI and TF methods are worth to mention that more than one cycle is needed in order to reconstruct power frequency currents using integration and larger memory locations are needed.

The terminal voltage of RC can be processed in order to reconstruct the original signal at its primary side. The input signal is reconstructed with compensating the output voltage of the RC [79]. The main objective of this project is to achieve accurate measurements with the advantage of reduced time for correct tracking. If we can increase the number of turns of the coil, we can increase the accuracy of the output signal. The numerical differentiation as a reconstruction method providing accurate as well as reduced time for correctly tracking the measured current. Requirement is within few samples to achieve the successful reconstruction. Mainly this paper to compare the accuracy and error detection between Reconstruction methods as Analog integrator and Numerical differentiation method [10]. 


\section{Experimental model and description}



Fig. 1: Block Diagram Representation of Reconstruction Methods.

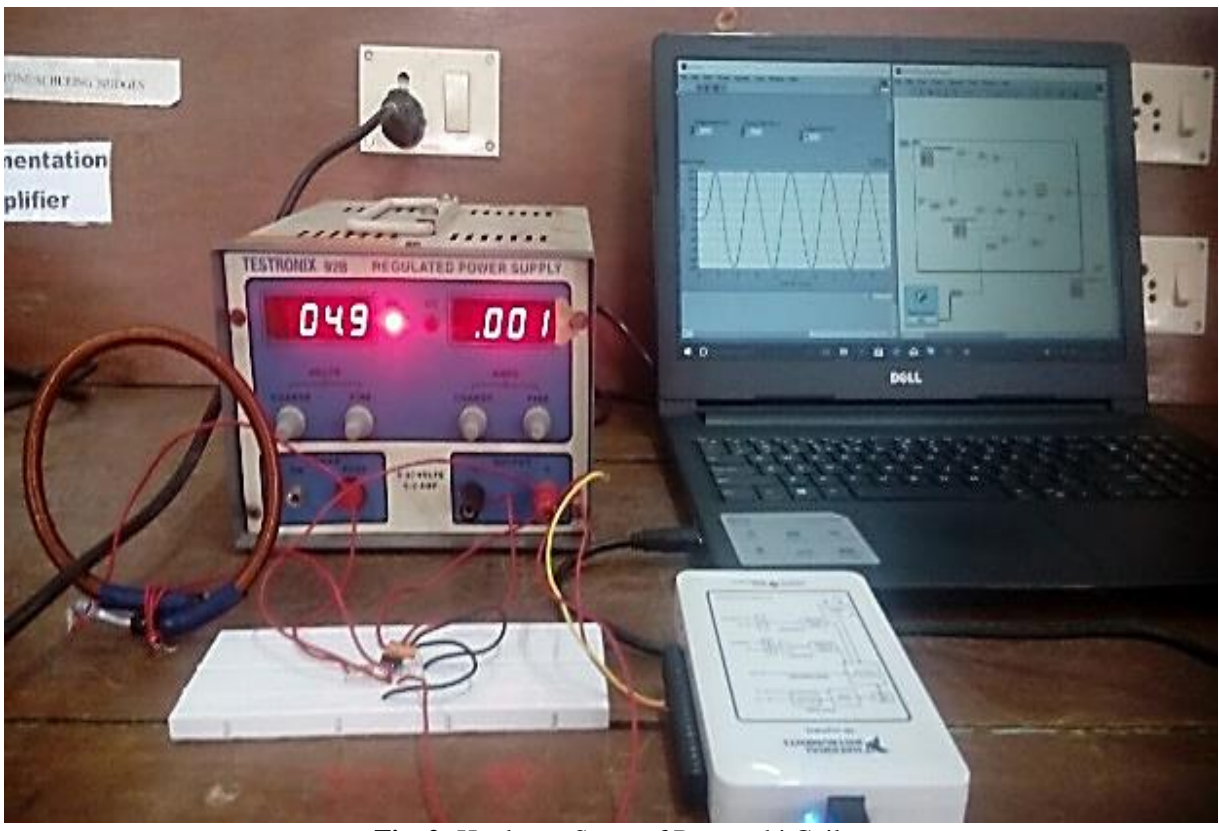

Fig. 2: Hardware Setup of Rogowski Coil.

The block diagram of $\mathrm{RC}$ reconstruct circuit is shown in Figure. 1 and hardware setup of RC is shown in Figure. 2. In RC, 31 SWG copper coils is used winding around the non-magnetic core. The diameter of the coil is measured using the screw gauge. The coil has 1000 turns, length of $420 \mathrm{~mm}$ length, $10 \mathrm{~mm}$ diameter, and cross-sectional area of $33^{*} 10^{\wedge} 3 \mathrm{~mm}^{2}$. An integrator is used in measurement and a control application is an element whose output signal is the time integral of its input signal. The Integrator has Resistance of $50 \mathrm{ohms}$ and Capacitance of 1 microfarad.

$\mathrm{RC}$ is centred on the conductor in order to sense its magnetic field. To evaluate the performance of RC, a commercial clamp ammeter and CT is used to obtain the reference current values. The induced voltage in the coil flows through the integrator circuit implemented in breadboard. The integrator output voltage is 1000 times proportional to the current passing through the conductor. And then the induced emf in coil is transferred to the differentiator setup in PC via DAQ Card. DAQ Card is an NI data acquisition device, used to interface the device output signal into the LabVIEW soft-

ware. And LabVIEW is used to differentiate the output voltage of the RC using Eq. (1) [8].

$\mathrm{e}(\mathrm{t})=-\mathrm{MI}_{\mathrm{m}} \omega \cos (\omega \mathrm{t} \pm \emptyset)(1)$

Where, $\mathrm{e}(\mathrm{t})$-EMF produced in the Coil

M-Mutual Inductance,

$\mathrm{I}_{\mathrm{m}}$-Amplitude Current,

$\omega$-Angular Frequency,

$\mathrm{t}$ - Time in sec,

$\emptyset$ - Phase Angle.

Finally, the reconstructed sinusoidal AC signal is produced in the LabVIEW output waveform graph. The reconstructed output values are shown in the Table. 1. RC output values and reference current values are compared in this table. The LabVIEW differentiator setup and output waveform from the LabVIEW software is shown in Figure.3.

\section{Results and discussions}

Table 1: Reconstructed Output Values and Reference Values

\begin{tabular}{llllll}
\hline S. No & $\begin{array}{l}\text { CT Output Current Val- } \\
\text { ues (A) }\end{array}$ & $\begin{array}{l}\text { RC Output } \\
(\mathrm{mV})\end{array}$ & $\begin{array}{l}\text { Proportional Current Val- } \\
\text { ues (A) }\end{array}$ & $\begin{array}{l}\text { RMS values of the CT Cur- } \\
\text { rent (A) }\end{array}$ & $\begin{array}{l}\text { RMS values of the RC Cur- } \\
\text { rent (A) }\end{array}$ \\
\hline 1 & 121.5 & 0.120 & 120 & 85.9 & 84.84 \\
2 & 162 & 0.160 & 160 & 114.534 & 113.12 \\
3 & 175 & 0.171 & 171 & 123.725 & 120.897 \\
4 & 205 & 0.200 & 200 & 144.935 & 141.4 \\
5 & 220 & 0.215 & 215 & 155.54 & 152.005 \\
6 & 240 & 0.235 & 235 & 169.68 & 166.145 \\
7 & 260 & 0.256 & 256 & 183.82 & 180.99 \\
8 & 270 & 0.265 & 265 & 190.89 & 187.355 \\
\hline
\end{tabular}




\begin{tabular}{llllll}
\hline 9 & 295 & 0.290 & 290 & 208.565 & 205.03 \\
10 & 350 & 0.343 & 343 & 247.45 & 242.501 \\
\hline
\end{tabular}

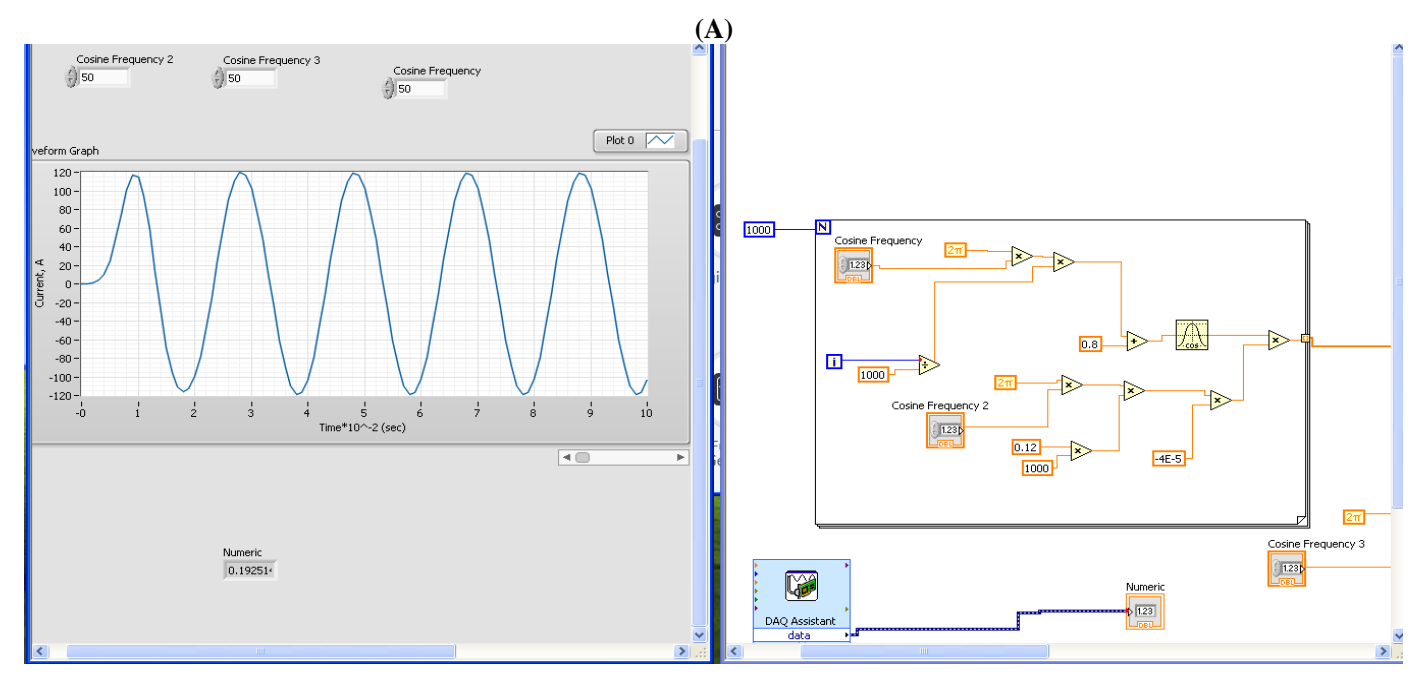

(B)

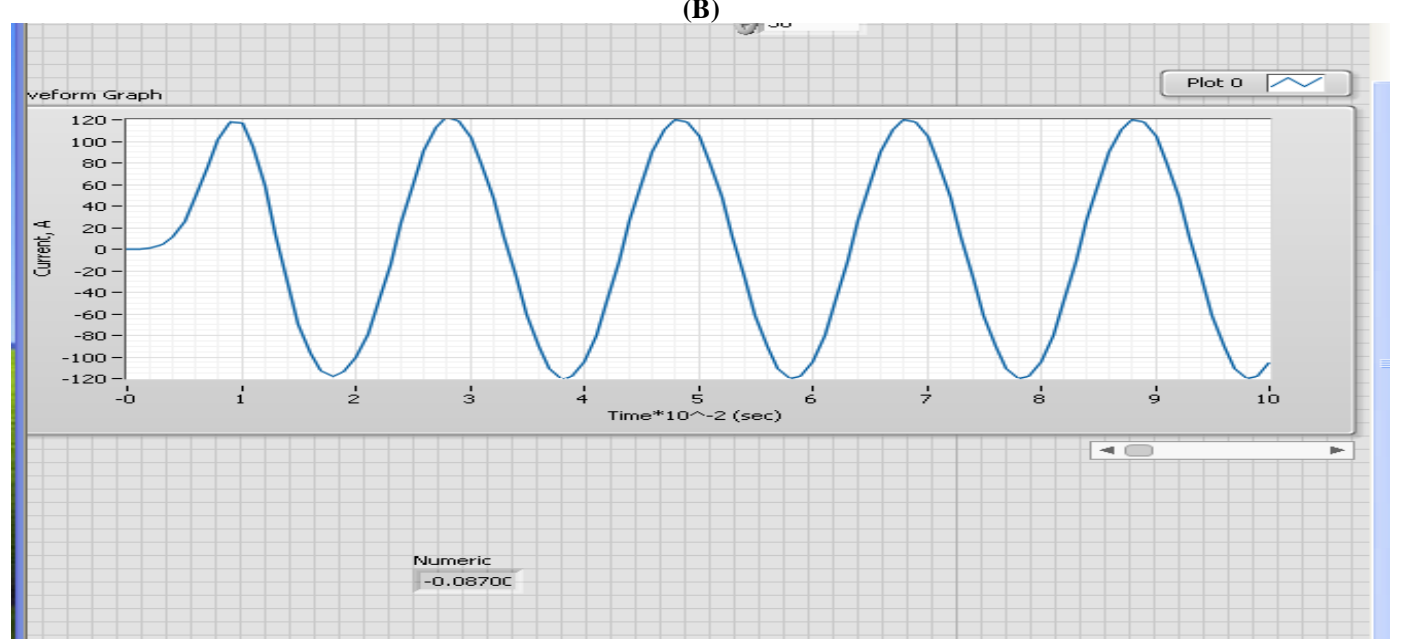

Fig. 3: Output Waveform of A) Reconstructed Current B) Reference Current.

An utilised reconstruction method for power frequency alternating current based on ND method has been presented. Its principle and construction has been discussed. Also, an experimental setup has been used for experimental validation of the proposed method The validity of this method is verified over a wide range of currents using RC design. Initially $122 \mathrm{~A}$ current passes through the conductor. RC senses this current in terms of the voltage (emf). The RC produced by emf is 1000 times proportional to the current passes through the conductor. Then the emf passes to the integrator and differentiator circuits. Produced emf is proportional to the current passing through the conductor. The RC senses the initial current value as $120 \mathrm{~A}$. And the sinusoidal output waveform produced in the LabVIEW. When compared to the CT and other devices $\mathrm{RC}$ gives the more accurate value of current. Time taken for the one complete cycle is around $20 \mathrm{msec}$. Applications of RC is power measurement, switchboards, thyristors, switching measurements, control signal display [11-13].

\section{Conclusion}

In this proposed method, we can achieve the simplest and efficient current measurement process. EMF produced in the coil is directly interfaced with the LabVIEW program using DAQ Card. So, it automatically receives the real-time values. And it takes the time period of $20 \mathrm{msec}$ for one complete cycle. We can measure the current value with less time period. From the analysis, errors in magnitude and phase angle of the power frequency $\mathrm{AC}$ reconstructed current depend on the RC design. Hence, these errors increase with the decrease in coil turns. The proposed differential reconstruction method succeeds in power frequency AC current reconstruction after 25 samples. The performance of the proposed method is not affected by the RC design, in the cases of step increase and a step decrease in measured current, the validity of the proposed method is verified with the same advantage of fast correct tracking. RC applications are power measurements, fault detection in transmission line and in electrical machines, switching measurements, arc melting furnaces, testing laboratories, industrial measurement instruments, unconventional current measuring systems and compare with analog integrator reconstruction method, numerical differential reconstruction method is the efficient method.

\section{References}

[1] Yu D. C., Cummins J. C., Z. Wang, H. J. Yoon and L. A. Kojovic, 2001 "Correction of current transformer distorted secondary currents due to saturation using artificial neural networks", IEEE Transaction. Power Delivery. 16 (2), pp. 189-194. https://doi.org/10.1109/61.915481.

[2] Hajipour E., Vakilian M., and Sanaye-Pasand M., 2015 "CurrentTransformer Saturation Compensation for Transformer Differential Relays", IEEE Transaction. Power Delivery. 30 (5), pp. 2293-2302. https://doi.org/10.1109/TPWRD.2015.2411736.

[3] Hemmati E., Shahrtash S. M., Jan. 2013 "Digital Compensation of Rogowski Coil's Output Voltage," IEEE Transaction. Instrumentation. and Measurement., 62 (1), pp. 71-82. https://doi.org/10.1109/TIM.2012.2212502. 
[4] Metwally I. A., Jan. 2014 "Novel designs of wideband Rogowski coils for high pulsed current measurement," IET Science. Measurement and Technology. 8 (1), pp. 9-16. https://doi.org/10.1049/iet-smt.2013.0045.

[5] Kojovic L. A., 2006, "Application of Rogowski coils used for protective relaying purposes," IEEE Transaction. Instrumentation and Measurement.

Pp.538-543. https://doi.org/10.1109/PSCE.2006.296371.

[6] Mohammad Hamed Samimi, Arash Mahari, Mohammad Ali Farahnakian, Hossein Mohseni, Year: 2015, "A Review on the RCPrinciples and Applications" IEEE Sensors Journal, 15 (2), Pages: 651 - 658. https://doi.org/10.1109/JSEN.2014.2362940.

[7] Chiampi M., Crotti G., and Morando A., Mar.2011 "Evaluation of Flexible RCPerformances in Power Frequency Application", IEEE Transaction. Instrumentation and Measurement. 60 (3), pp.854 862. https://doi.org/10.1109/TIM.2010.2060223.

[8] Mohamed E. Ibrahim, and Amr M. Abd-Elhady, Year: 2016, "Differential Reconstruction Method for Power Frequency AC Current Measurement Using RC'IEEE Sensors Journal, 16 (23), Pages: 8420 - 8425.

[9] El_Kalashy N. I., Kawady T. A., Ismail E. M., and Taalab A. I., 2016 "Fundamental current phasor tracking using DFT extraction of RC signal", IET-Science Measurement and Technology, 10 (4), pp. 296-305.

[10] Hong Y. Y. In addition, Chang-Chian P. C., 2008 "Detection and correction of distorted current transformer current using wavelet transform and artificial intelligence", Process and Instrumentation Engineering. Technol., Generation. Transmission and Distribution. Two, pp. 566575.

[11] Ashok Kumar L; Indragandhi V; Sujith Kumar N Design and implementation of single-phase inverter without transformer for PY applications, IET Renewable Power Generation, 2018, Vol. 12 Iss. Five, pp. 547-554.

[12] T. Arunkumari, V. Indragandhi "An overview of high voltage conversion ratio DC-DC converter configurations used in DC microgrid architectures", Renewable and Sustainable Energy Reviews, Vol. 77, pp. 670-687, 2017 https://doi.org/10.1016/j.rser.2017.04.036.

[13] R. Raja Singh, B. Anil Kumar, D. Shruthi, Ramraj Panda, C. Thanga Raj, "Review and experimental illustrations of electronic load controller used in standalone Micro-Hydro generating plants," Engineering Science and Technology, an International Journal, Elsevier, 2018 